\title{
Limited Influence of Flail Chest in Patients With Blunt Thoracic Trauma - A Matched-pair Analysis
}

\author{
PETER GETZ, PHILIPP MOMMSEN, JAN-DIERK CLAUSEN and MARCEL WINKELMANN
}

Trauma Department, Hannover Medical School, Hannover, Germany

\begin{abstract}
Background: Flail chest is considered as one of the most severe forms of blunt thoracic trauma. However, its actual influence on post-traumatic morbidity and mortality is debatable. Materials and Methods: A retrospective cohort analysis was performed of multiply injured patients (injury severity score $\geq 16$ ) at a level I trauma center. Flail chest was defined as segment fracture of at least three consecutive ribs on at least one side. Propensity score matching was performed. Results: A total of 600 patients were included, with a mean age of $44.1 \pm 19.1$ years and a mean injury severity score of 31.6 \pm 10.4 . Overall, 367 patients (61.2\%) had a serial rib fracture. Forty-five patients (7.5\%) presented with flail chest. Patients with flail chest more often had lung contusions $(70$ vs. $50 \%, p=0.04$ ) and pneumo-/hematothorax (93 vs. $71 \%$, $p=0.005)$. There were no differences in post-traumatic morbidity and mortality. Conclusion: Flail chest had no independent influence in addition to injury severity on posttraumatic morbidity and mortality in multiply injured patients with blunt thoracic trauma.
\end{abstract}

Blunt trauma is the leading cause of thoracic injuries. About $50 \%$ of all multiply injured patients suffer from blunt chest trauma (1). It is the second most common severely injured body region [abbreviated injury scale (AIS) >3] in Germany after the head (2). According to recent research, chest trauma is responsible for about $25 \%$ of all deaths in multiply injured patients $(1,3,4)$. Blunt thoracic trauma thereby causes higher morbidity and mortality compared to multiply injured patients without chest trauma (5-8). Therefore, early and

This article is freely accessible online.

Correspondence to: Marcel Winkelmann, MD, Trauma Department, Hannover Medical School, Carl-Neuberg-Strasse 1, D-30625 Hannover, Germany. Tel: +49 17615322684, e-mail: winkelmann. marcel@mh-hannover.de

Key Words: Multiple injuries, thoracic trauma, serial rib fracture, flail chest, acute respiratory distress syndrome. precise diagnose of specific chest injuries is useful for further clinical treatment in order to avoid posttraumatic complications and finally to reduce mortality.

In particular, flail chest as a severe form of a serial rib fracture should be deemed to be a predictor of morbidity and mortality (9). Its incidence in multiply injured patients varies from 1 to $16 \%$ among different studies, with reported mortality rates up to $25 \%$ (10-13). Flail chest can lead to chest wall instability and results in respiratory insufficiency with major difficulties in clinical treatment. The need for intubation and mechanical ventilation can cause ventilator-associated pneumonia and contribute to high rates of mortality in patients with flail chest (14). Serial rib fractures and flail chest are frequently accompanied by lung contusions, averaging 46-54\% (11, $12,15)$. Contusions of the lung, as well as chest wall instabilities are known to be a trigger for significantly high rates of complications and mortality. Experimental studies by Capello et al. demonstrated that lung contusions have a more negative effect on respiratory insufficiency than chest wall instability itself (16). In particular, the combination of flail chest and lung contusions lead to notably higher rates of mortality, reported to be as high as $40 \%(11,17)$. While isolated unilateral flail chest has a mortality rate less than $6 \%$, accompanying injuries substantially increase mortality (9).

This raises the question whether flail chest has an independent impact on outcome or is just an indicator of increased severity of chest injury $(4,7,14,18)$. Moreover, several studies have focused on the effect of single rib fractures on morbidity and mortality and thereby neglected specific defined fracture patterns like flail chest or compared mortality and morbidity between younger and older patients (wide range of patients) $(10,19-21)$. In addition, the definition of flail chest is largely inconsistent. The most accepted definition, which is applied in this study, is a segmental fracture of three or more consecutive ribs (22). However, different studies use the terms flail chest and unstable chest with inverted breathing interchangeably. Thus, the impact and prognostic value of flail chest are still a topic of controversial discussion (7, 15, 21-25). 
Therefore, this study aimed to analyze the independent influence of precisely defined flail chest on post-traumatic morbidity [acute respiratory distress syndrome (ARDS), ventilator-associated pneumonia (VAP), prolonged mechanical ventilation etc.] and mortality in multiply injured patients with relevant blunt chest trauma.

\section{Materials and Methods}

A retrospective cohort analysis was performed. Between January 2005 and December 2014, all multiply injured patients [injury severity score (ISS) $\geq 16$ ] with a minimum age of 15 years primarily admitted to a single level I trauma center in a midEuropean metropolitan area were included (26). The study was approved by the Institutional Review Board (No. 3392-2016). All patients underwent precisely defined trauma management according to current guidelines. Demographic and baseline data including age, sex, mortality, duration of mechanical ventilation, duration of intensive care as well as overall in-patient care, and transfusion requirements [packed red blood cells (PRBC), fresh frozen plasma (FFP) and platelet concentrate (PC)] were collected from the hospital information system. Injury pattern and organrelated severity were classified using the 2008 update of the abbreviated injury scale (AIS) (22). Overall severity of injury was calculated using the ISS. Systolic blood pressure (SBP; in $\mathrm{mmHg}$ ) was measured by either oscillometric method or, if applicable, invasive arterial measurement. SBP and heart rate (HR) were used to calculate the shock index (SI=HR/SBP). A shock index between 0.5 and 0.7 is considered physiological (27). Shock at admission was defined as SBP $\leq 90 \mathrm{mmHg}$ with/without shock index $\geq 0.9$. Multiple organ dysfunction syndrome (MODS) was diagnosed on the basis of MODS score by Marshall et al. MODS was deemed present if the sum of single organ dysfunctions was $>8$ on at least one day (28).

All patients underwent whole-body computed tomography (CT) with contrast agent administration at the time of admission. Appraisal of CT scans were performed by a consultant with specialization in skeletal radiology. All CT scans were checked for rib fractures and lung contusions, as well as pneumo-and hematothorax. A serial rib fracture was present if three consecutive ribs were fractured on at least one side. A flail chest was defined as segment fractures of at least three consecutive ribs on at least one side (22).

Statistical analyses were performed with IBM SPSS (Version 23; IBM, Armonk, NY, USA). Gaussian distributed variables were analyzed using parametric tests (Student's $t$-test) and other variables were analyzed using non-parametric tests (MannWhitney test for independent data; Wilcoxon test for dependent data). Fisher's exact test (exact chi-squared test) was used in the analysis of contingency tables. Binomial logistic regression analyses were performed and odds ratios (OR) and $95 \%$ confidence intervals $(95 \% \mathrm{CI})$ were calculated. In order to avoid a potential bias due to different overall injury severity or injury pattern in patients with and without flail chest, a matched-pair analysis was performed using propensity score matching with nearest-neighbor matching and a 1:3 match ratio. The following covariates and flail chest as binary treatment indicator were used: age, sex, ISS, $\mathrm{AIS}_{\text {Head }}, \mathrm{AIS}_{\text {Face }}$, AIS $\mathrm{S}_{\text {Abdomen }}$, AIS $\mathrm{Extremities}_{\text {, AIS }} \mathrm{External}_{\text {. The }}$ significance level was set at $p=0.05$.

\section{Results}

A total of 972 patients were evaluated for eligibility: 600 patients fulfilled the inclusion criteria and were enrolled for further statistical analysis (see also Figure 1). The mean follow-up was $23.9 \pm 20.1$ days. Mean age was $44.1 \pm 19.1$ years and mean ISS was $31.6 \pm 10.4 ; 439$ patients $(73.2 \%)$ were male. Table I summarizes the demographic, baseline and outcome data.

Serial rib fractures were diagnosed in 367 patients $(61.2 \%)$, of which 45 (7.5\%) presented with flail chest. There was no difference between patients with and without serial rib fractures regarding ISS. Although mortality was increased in the group with serial rib fracture (16.6 vs. $10.3 \%, p=0.03$ ), the presence of a serial rib fracture was not identified as an independent predictor of mortality in a multivariate regression analysis $(\mathrm{OR}=1.2,95 \% \mathrm{CI}=0.7-2.2$; $p=0.5$ ). Pneumo-and hematothorax were significantly more frequent in patients with serial rib fractures (74.1 vs. 58.8\%, $p<0.001)$. However, there were no differences in occurrence of lung contusions ( $58.0 \mathrm{vs} .59 .7 \%, p=0.7)$. There were no differences in frequency of ARDS (34.3vs. $34.3 \%, p=1.0$ ) or VAP (16.3 vs. $11.2 \%, p=0.09)$. Thus, the duration of mechanical ventilation $(298 \pm 351 v s .229 \pm 278 \mathrm{~h}, p=0.3)$ and intensive care $(16.0 \pm 15.8 v s .13 .3 \pm 12.7$ days, $p=0.2)$ did not differ significantly between the two groups.

One hundred and fifty patients (40 flail chest $v s$. 110 serial rib fracture but no flail chest) were matched using a propensity score matching with nearest-neighbor matching and a 1:3 match ratio to reduce confounding effects. Table II gives an overview of their demographic, baseline, and clinical data. Regarding matching quality, there were no significant differences in age $(52.7 \pm 19.1 \mathrm{vs}$. 53.4 \pm 18.3 years, $p=0.9$ ), gender distribution, injury severity (ISS 30.7 $\pm 9.5 \mathrm{vs}$. $28.8 \pm 8.4, p=0.3$ ) or injury pattern (see also Table II). Patients with flail chest more often suffered from lung contusion (70 vs. 50\%, $p=0.04$ ) and pneumo-/hematothorax (93 vs. 71\%, p=0.005). Mortality was also increased (18vs. $7 \%, p=0.1)$. However, this did not reach significance. Focusing on post-traumatic complications, patients with flail chest did not suffer from ARDS (38vs. 33\%, $p=0.7$ ) or VAP (23 vs. 16\%, $p=0.5$ ) more often. The same applied to duration of mechanical ventilation $(371 \pm 403 v s .288 \pm 358 \mathrm{~h}$, $p=0.2)$ and duration of intensive care $(19.8 \pm 17.6 \mathrm{vs}$. $15.5 \pm 15.2$ days, $p=0.2$ ).

\section{Discussion}

The purpose of this study was to analyze the independent influence of flail chest on morbidity and mortality in multiply injured patients with relevant blunt thoracic trauma. Although flail chest was associated with a more frequent presence of lung contusion and pneumo-/hematothorax, no 


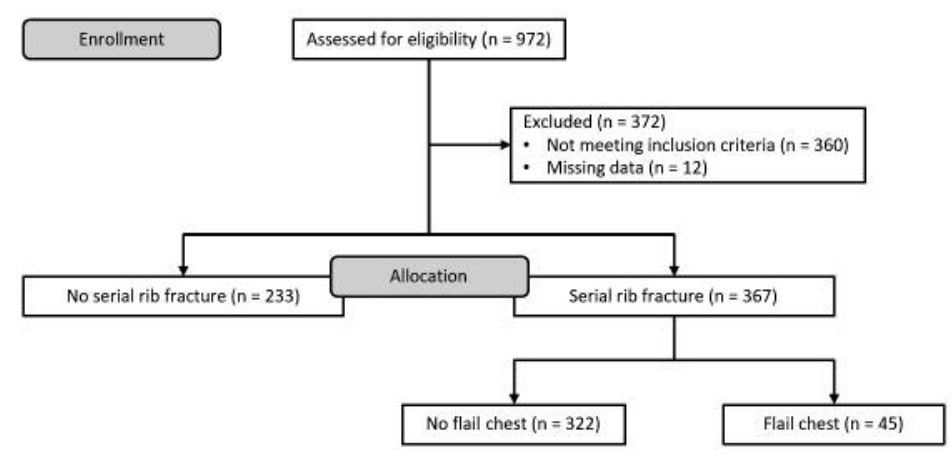

Figure 1. Flow diagram of study patients.

Table I. Demographic, baseline and clinical data of the population, as well as patients with and without serial rib fracture.

\begin{tabular}{|c|c|c|c|c|}
\hline Characteristic & Total $(n=600)$ & Serial rib fracture $(n=367)$ & No serial rib fracture $(n=233)$ & $p$-Value \\
\hline Age (years), mean \pm SD & $44.1 \pm 19.1$ & $49.5 \pm 18.6$ & $35.6 \pm 16.7$ & $<0.001^{\mathrm{a}}$ \\
\hline Male gender, n (\%) & $439(73.2)$ & $261(71.1)$ & $178(76.4)$ & $0.2^{\mathrm{b}}$ \\
\hline Injury severity score, mean \pm SD & $31.6 \pm 10.4$ & $31.6 \pm 10.8$ & $31.7 \pm 9.8$ & $0.7^{\mathrm{a}}$ \\
\hline \multicolumn{5}{|l|}{ Abbreviated injury scale, mean $\pm S D$} \\
\hline Head & $2.0 \pm 1.7$ & $1.8 \pm 1.7$ & $2.1 \pm 1.7$ & $0.054^{\mathrm{a}}$ \\
\hline Face & $0.8 \pm 1.1$ & $0.8 \pm 1.1$ & $0.8 \pm 1.2$ & $0.7^{\mathrm{a}}$ \\
\hline Thorax & $3.6 \pm 0.7$ & $3.7 \pm 0.7$ & $3.5 \pm 0.7$ & $0.001^{\mathrm{a}}$ \\
\hline Abdomen & $1.4 \pm 1.5$ & $1.5 \pm 1.5$ & $1.2 \pm 1.5$ & $0.02^{\mathrm{a}}$ \\
\hline Extremities & $2.3 \pm 1.3$ & $2.2 \pm 1.3$ & $2.3 \pm 1.3$ & $0.1^{\mathrm{a}}$ \\
\hline External & $0.8 \pm 0.9$ & $0.8 \pm 0.9$ & $0.9 \pm 1.0$ & $0.3^{\mathrm{a}}$ \\
\hline Lung contusion, $\mathrm{n}(\%)$ & $352(58.7)$ & $213(58.0)$ & $139(59.7)$ & $0.7^{b}$ \\
\hline Pneumo-/hematothorax, n (\%) & $409(68.2)$ & $272(74.1)$ & $137(58.8)$ & $<0.001^{\mathrm{b}}$ \\
\hline ARDS, n (\%) & $206(34.3)$ & $126(34.3)$ & $80(34.3)$ & $1.0^{\mathrm{b}}$ \\
\hline VAP, n $(\%)$ & $86(14.3)$ & $60(16.3)$ & $26(11.2)$ & $0.09^{\mathrm{b}}$ \\
\hline Duration of mechanical ventilation (h), mean $\pm \mathrm{SD}$ & $271 \pm 326$ & $298 \pm 351$ & $229 \pm 278$ & $0.3^{\mathrm{a}}$ \\
\hline Duration of intensive care (days), mean \pm SD & $15.0 \pm 14.7$ & $16.0 \pm 15.8$ & $13.3 \pm 12.7$ & $0.2^{\mathrm{a}}$ \\
\hline Duration of in-patient care (days), mean \pm SD & $23.9 \pm 20.1$ & $24.0 \pm 19.7$ & $23.7 \pm 20.8$ & $0.8^{\mathrm{a}}$ \\
\hline \multicolumn{5}{|l|}{ Transfusion requirement } \\
\hline PRBC 48-h (units), mean \pm SD & $7.7 \pm 10.9$ & $8.3 \pm 11.5$ & $6.9 \pm 10.0$ & $0.2^{\mathrm{a}}$ \\
\hline FFP 48 -h (units), mean \pm SD & $6.0 \pm 8.8$ & $6.3 \pm 8.7$ & $5.5 \pm 8.9$ & $0.1^{\mathrm{a}}$ \\
\hline $\mathrm{PC}$ 48-h (units), mean \pm SD & $1.0 \pm 2.0$ & $1.1 \pm 2.1$ & $0.8 \pm 1.9$ & $0.08^{\mathrm{a}}$ \\
\hline $\mathrm{PRBC}$ (units), mean $\pm \mathrm{SD}$ & $13.5 \pm 17.1$ & $14.2 \pm 17.2$ & $12.3 \pm 16.8$ & $0.1^{\mathrm{a}}$ \\
\hline FFP (units), mean \pm SD & $8.7 \pm 13.4$ & $9.1 \pm 12.7$ & $8.0 \pm 14.4$ & $0.1^{\mathrm{a}}$ \\
\hline $\mathrm{PC}$ (units), mean $\pm \mathrm{SD}$ & $1.4 \pm 3.6$ & $1.6 \pm 3.7$ & $1.2 \pm 3.4$ & $0.049^{\mathrm{a}}$ \\
\hline Mortality, n (\%) & $85(14.2)$ & $61(16.6)$ & $24(10.3)$ & $0.03^{\mathrm{b}}$ \\
\hline Shock, n (\%) & $218(36.3)$ & $135(36.8)$ & 83 (35.6) & $0.8^{\mathrm{b}}$ \\
\hline
\end{tabular}

ARDS: Acute respiratory distress syndrome, FFP: fresh-frozen plasma, PC: platelet cells, PRBC: packed red blood cells, SD: standard deviation,

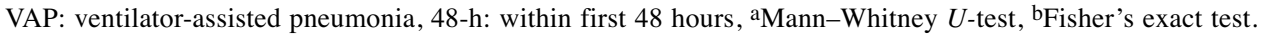

independent influence on post-traumatic course of treatment, including occurrence of ARDS as well as mortality, was found.

This might, to some extent, be in contrast to the clinical reality. From daily experience, patients with a flail chest injury do have a more complicated clinical course. However, there are two major concerns. For one thing, there are several different definitions of flail chest and for another, flail chest could be a surrogate parameter of the severity of organrelated injury.

Addressing the first concern in depth, flail chest is more a collective term rather than a firm definition. The most accepted definition is most probably a segmental fracture of three or more consecutive ribs. This is consistent with the AIS. The Association for the Advancement of Automotive Medicine defines flail chest as three or more adjacent ribs, 
in vivo $33: 133-139(2019)$

Table II. Demographic, baseline and clinical data of patients with and without flail chest after propensity score matching.

\begin{tabular}{|c|c|c|c|}
\hline Characteristic & Flail chest $(n=40)$ & No flail chest $(n=110)$ & $p$-Value \\
\hline Age (years), mean $\pm \mathrm{SD}$ & $52.7 \pm 19.1$ & $53.4 \pm 18.3$ & $0.9^{\mathrm{a}}$ \\
\hline Male gender, $\mathrm{n}(\%)$ & $27(68)$ & $78(71)$ & $0.7^{\mathrm{b}}$ \\
\hline Injury Severity Score (ISS), mean \pm SD & $30.7 \pm 9.5$ & $28.8 \pm 8.4$ & $0.3^{\mathrm{a}}$ \\
\hline \multicolumn{4}{|l|}{ Abbreviated injury scale, mean $\pm \mathrm{SD}$} \\
\hline Head & $1.7 \pm 1.6$ & $1.7 \pm 1.6$ & $0.9^{\mathrm{a}}$ \\
\hline Face & $0.7 \pm 1.0$ & $0.7 \pm 1.0$ & $0.9^{\mathrm{a}}$ \\
\hline Thorax & $3.9 \pm 0.8$ & $3.7 \pm 0.7$ & $0.3^{\mathrm{a}}$ \\
\hline Abdomen & $1.2 \pm 1.4$ & $1.1 \pm 1.3$ & $1.0^{\mathrm{a}}$ \\
\hline Extremities & $2.4 \pm 1.0$ & $2.2 \pm 1.0$ & $0.5^{\mathrm{a}}$ \\
\hline External & $0.7 \pm 0.8$ & $0.7 \pm 0.8$ & $0.8 \mathrm{a}$ \\
\hline Lung contusion, $\mathrm{n}(\%)$ & $28(70)$ & $55(50)$ & $0.04^{\mathrm{b}}$ \\
\hline Pneumo-/hematothorax, n (\%) & $37(93)$ & $78(71)$ & $0.005^{\mathrm{b}}$ \\
\hline ARDS, n $(\%)$ & $15(38)$ & $36(33)$ & $0.7^{\mathrm{b}}$ \\
\hline $\mathrm{VAP}, \mathrm{n}(\%)$ & $9(23)$ & $18(16)$ & $0.5^{\mathrm{b}}$ \\
\hline Duration of mechanical ventilation $(\mathrm{h})$, mean \pm SD & $371 \pm 403$ & $288 \pm 358$ & $0.2^{\mathrm{a}}$ \\
\hline Duration of intensive care (days), mean $\pm \mathrm{SD}$ & $19.8 \pm 17.6$ & $15.5 \pm 15.2$ & $0.2^{\mathrm{a}}$ \\
\hline Duration of in-patient care (days), mean $\pm \mathrm{SD}$ & $29.2 \pm 24.2$ & $23.1 \pm 16.4$ & $0.3^{\mathrm{a}}$ \\
\hline \multicolumn{4}{|l|}{ Transfusion requirement } \\
\hline PRBC 48-h (units), mean \pm SD & $7.8 \pm 9.4$ & $6.4 \pm 11.7$ & $0.08^{\mathrm{a}}$ \\
\hline FFP 48-h (units), mean \pm SD & $5.3 \pm 6.7$ & $4.7 \pm 7.7$ & $0.3^{\mathrm{a}}$ \\
\hline $\mathrm{PC} 48$-h (units), mean \pm SD & $0.9 \pm 1.8$ & $0.8 \pm 1.9$ & $0.7^{\mathrm{a}}$ \\
\hline $\mathrm{PRBC}$ (units), mean $\pm \mathrm{SD}$ & $13.6 \pm 19.7$ & $11.3 \pm 16.7$ & $0.4^{\mathrm{a}}$ \\
\hline FFP (units), mean \pm SD & $7.7 \pm 13.5$ & $7.7 \pm 12.6$ & $0.6^{\mathrm{a}}$ \\
\hline $\mathrm{PC}$ (units), mean $\pm \mathrm{SD}$ & $2.0 \pm 6.1$ & $1.4 \pm 4.4$ & $0.7^{\mathrm{a}}$ \\
\hline Mortality, n (\%) & $7(18)$ & $8(7)$ & $0.1^{\mathrm{b}}$ \\
\hline Shock, n (\%) & $10(25)$ & $32(29)$ & $0.7^{\mathrm{b}}$ \\
\hline
\end{tabular}

ARDS: Acute respiratory distress syndrome, FFP: fresh-frozen plasma, PC: platelet cells, PRBC: packed red blood cells, SD: standard deviation,

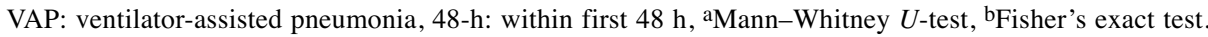

each fractured in more than one location (e.g. posterolateral and anterolateral) to create a free-floating segment, which may or may not result in paradoxical chest movement (22). Two major studies carried out by Dehghan et al. (11) and Schulz-Drost et al. (12) used the AIS coding to identify patients with flail chest. Other studies regarding flail chest either did not specify the definition applied (10) or used an umbrella term which left a wide margin of interpretation (9, 18). Borman et al. (9), as well as Kilic et al. (18) state that "flail occurs when the integrity of the thoracic rigid cage is compromised and a segment, its size depending on the number of fractured ribs or costal cartilages, loses its continuity with the rest of the thoracic cage and moves paradoxically during spontaneous respiration". This represents a clinically driven definition and is partially in contrast to the anatomic AIS definition. Paradoxical chest movement is a sine qua non in the one definition and optional in the other. Thus, comparability of patients is severely limited. It seems conclusive to clinically identify patients at particular risk. The use of the clinical definition therefore results in a more seriously ill patient group. Not surprisingly, these patients have a poorer outcome compared to clinically more stable ones. As yet there are no data about inter-and intra-rater reliability. Thus, comparability and generalizability of the findings are very limited. To exaggerate, the gain of knowledge is that seriously ill patients are more ill than healthier patients. Therefore, if the clinical definition of flail chest is unreliable, it might be better to use the anatomic definition. Major advantages are its objectivity and reproducibility. These advantages, however, are counteracted by the inherent problems of multicentric registry data. Hundreds and even thousands of different users enter data. In theory, all users have to apply the same definition of flail chest. In practice, there is no validation of the data. In the German Trauma Registry, there is only a consistency check. Entries are only spot-checked if documentation of diagnoses in the clinical information system is consistent with the documentation in the registry. The extent to which documented diagnoses are actually appropriate according to the AIS definition is simply not checked. Therefore, data validity of multicentric registries is questionable to some extent. Dehghan et al. identified 3,467 patients $(1 \%)$ with flail chest during a 3-year period using the US/Canadian National Trauma Data Bank (11). Schulz- 
Drost et al. identified 3,492 patients (16.1\%) with flail chest during a 5-year period using the German Trauma Registry (12). Both authors used the same AIS-based definition. The substantial difference in the incidence might be explained, to some extent, by different inclusion criteria. The US/Canadian National Trauma Data Bank includes every hospitalized patient with a relevant injury, whereas the German Trauma Registry includes patients admitted to the operating theatre as well as Intensive Care Unit following Emergency Room treatment. Dehghan et al. (11) included patients with an ISS of 9 or higher and Schulz-Drost et al. (12) with an ISS of 16 or higher. Against this background, incidence of flail chest in the study performed by Schulz-Drost et al. (16.1\%) (12) seems reasonable but is rather high compared to our own data $(7.5 \%)$. This is even more surprisingly since our own data are part of the German Trauma Registry, and demographic as well as baseline parameters are comparable for both patient populations. Therefore, the question arises again whether data validity exists. A major advantage of our study is its data validity. A CT scan was performed in all the patients. The diagnoses were verified by a consultant with special qualification in diagnostic radiography. In summary, the lack of a standardized definition and the uncertain data validity compromise the significance and generalizability of many studies.

However, despite definitional uncertainties, the more interesting question seems to be whether flail chest has an independent influence on morbidity and mortality or is just a surrogate parameter of the severity of organ-related injury. Velmahos et al. identified flail chest and extrathoracic injuries as independent risk factors for significant respiratory complications (14). Kilic et al. reported that injury severity was a major risk factor for mortality from flail chest trauma. However, this study seems to have serious shortcomings. The reported mean ISS of patients with traumatic brain injury and flail chest was 75 , which is equivalent to a non-survivable injury. Nevertheless, mortality was $19 \%$. Patients with flail chest without traumatic brain injury had a mean ISS of 55.7; the corresponding mortality was $0 \%$ (18). Based on our professional experience and with regard to the structure and validity of the ISS, these findings are inexplicable. Borman et al. reported that accompanying extra-thoracic injuries, especially traumatic brain injury, increased mortality. Since there was no control group, it is not clear whether this finding is only due to the increased ISS. The authors did not make a statement on the independent role of flail chest compared with other thoracic injuries (9). Huber et al. demonstrated that extra-thoracic factors (age over 65 years, blood transfusion, shock, severe extra-thoracic injury) prolong ventilation. This also applies to intrathoracic injuries. Nearly every type of injury to the lung parenchyma, hemo- and severe pneumothorax, and major thoracic vessel and cardiac injuries prolonged ventilation with a correlation of injury severity to the expected prolongation (29). Dehghan et al. also identified traumatic brain injury as a major risk factor of adverse outcome in patients with flail chest (11). In line with our own results, Huber et al. demonstrated unilateral flail chest not to be a predictor of increased mortality; only bilateral flail chest was significantly associated with elevated mortality (29). Since there were no patients with bilateral flail chest in our own study population, we are not able to make an objective statement concerning this matter. Schulz-Drost et al. reported a more frequent occurrence of multiple organ dysfunction syndrome and septic complications in patients with flail chest. Mortality was increased accordingly. Bilateral flail chest, as expected, had the highest mortality (12). However, injury severity was increased in the flail chest group $(35.1 \pm 14.2 \mathrm{vs}$. $28.3 \pm 11.3$ ) and the authors omitted analysis of the impact of flail chest injuries independent of increased injury severity (12). In summary, our own results support the assumption that flail chest is a surrogate parameter of increased severity of thoracic injury rather than an independent predictor of adverse outcome.

However, there are certain issues that are poorly analyzed or discussed. The impact of lung contusion and laceration is hardly understood yet. Huber et al. demonstrated a linear correlation of injury severity to the expected prolongation of ventilation for lung contusion as well as lung laceration (29). But these findings are inconsistent. Dehghan et al. reported a significant increase in the rate of pulmonary complications, even though the magnitude of the differences was low and clinical relevance was not existent (11). From a clinical point of view, it seems obvious that parenchymatous injuries to the lung affect ventilation and the risk of ARDS. But it is difficult to prove. What matters is the extent of lung contusion. However, this depends on the period from the accident to the first CT. The shorter the period, the greater the risk that the real extent of contusion is masked. To our knowledge, no study has yet focused on the correlation between volumetric extent of lung contusion and pulmonary complications. Another relevant issue is the possible bias caused by patients who died before reaching the hospital or in the Emergency Room. Kleber et al. reported that tension pneumothorax was one of the leading causes of traumarelated preventable deaths in the pre-hospital setting (30). Various studies and our own results show that pneumothorax is more common in patients with flail chest $(11,12,29)$. Since every study excluded patients who died pre-hospital or in the Emergency Room, it is unclear, to what extent this biases the result. It is conceivable that patients with especially severe thoracic injuries die before reaching hospital and only patients with a rather benign form of flail chest arrive still alive at the hospital. Due to this selection bias, the outcome differences may be levelled.

This limitation also applies to our retrospective study. However, data quality is high and definition of flail chest in 
comparison to serial rib fractures is well-defined. Nevertheless, there are some limitations one should take into consideration when interpreting the data. Therapy of blunt thoracic trauma has continuously changed during the study period. Alternating position and prone position replaced kinetic therapy. Respiratory therapy also changed. However, there are no convincing data that demonstrate advantages of one approach yet. There are other limitations that might bias our results. Eligibility criteria were well-defined; therefore, numerous patients were excluded. Furthermore, this study analyzed patients with blunt thoracic trauma only. Due to totally different dynamics of blunt and penetrating trauma, a transfer of results is not possible. Therefore, we cannot give any objective recommendations regarding penetrating trauma. However, our results can be generalized to high income countries with comparable therapy according to actual guidelines.

In conclusion, we demonstrated that there was no independent impact of flail chest on post-traumatic mortality and morbidity in multiply injured patients with blunt thoracic trauma. Therefore, it seems inappropriate to give recommendations on operative therapy based on the anatomic diagnosis of flail chest alone, although this is of particular interest. Future studies should analyze which particular injuries in blunt thoracic trauma independently worsen the outcome and necessitate an operative therapy. Special attention needs to be paid to the extent of lung contusion and in particular fracture patterns that hamper ventilation. A set definition of flail chest and scientifically sound knowledge about its impact on multiply injured patients provides the basis for this and helps to draw conclusions about the potential benefits of an operative therapy.

\section{References}

1 Galan G, Penalver JC, Paris F, Caffarena JM, Jr., Blasco E, Borro JM, Garcia-Zarza A, Padilla J, Pastor J and Tarrazona V: Blunt chest injuries in 1696 patients. Eur J Cardiothorac Surg 6(6): 284-287, 1992.

2 German Trauma Society (DGU) Committee on Emergency Medicine: Annual report Trauma Register (TR-DGU) 2017. Available from: http://www.traumaregister-dgu.de/fileadmin/ user_upload/traumaregister-dgu.de/docs/Downloads/TRDGU_Annual_Report_2017.pdf Last accessed 30.01.2018.

3 El-Menyar A, Latifi R, AbdulRahman H, Zarour A, Tuma M, Parchani A, Peralta R and Al Thani H: Age and traumatic chest injury: A 3-year observational study. Eur J Trauma Emerg Surg 39(4): 397-403, 2013.

4 Ziegler DW and Agarwal NN: The morbidity and mortality of rib fractures. J Trauma 37(6): 975-979, 1994.

5 Waydhas C, Nast-Kolb D, Trupka A, Jochum $M$ and Schweiberer L: Die bedeutung des traumatisch-hämorrhagischen schocks und der thoraxverletzung für die prognose nach polytrauma. Hefte Unfallheilkd 212: 104-105, 1990.

6 Stellin G: Survival in trauma victims with pulmonary contusion. Am Surg 57(12): 780-784, 1991.
7 Clark GC, Schecter WP and Trunkey DD: Variables affecting outcome in blunt chest trauma: Flail chest vs. Pulmonary contusion. J Trauma 28(3): 298-304, 1988.

8 Pape HC, Regel G, Dwenger A, Sturm JA and Tscherne H: Influence of thoracic trauma and primary femoral intramedullary nailing on the incidence of ards in multiple trauma patients. Injury 24(Suppl 3): S82-103, 1993.

9 Borman JB, Aharonson-Daniel L, Savitsky B, Peleg K and Israeli Trauma G: Unilateral flail chest is seldom a lethal injury. Emerg Med J 23(12): 903-905, 2006.

10 Sirmali M, Turut H, Topcu S, Gulhan E, Yazici U, Kaya S and Tastepe I: A comprehensive analysis of traumatic rib fractures: Morbidity, mortality and management. Eur J Cardiothorac Surg 24(1): 133-138, 2003.

11 Dehghan N, de Mestral C, McKee MD, Schemitsch EH and Nathens A: Flail chest injuries: A review of outcomes and treatment practices from the national trauma data bank. J Trauma Acute Care Surg 76(2): 462-468, 2014.

12 Schulz-Drost S, Oppel P, Grupp S, Krinner S, Langenbach A, Lefering $\mathrm{R}$ and Mauerer A: Bony injuries of the thoracic cage in multiple trauma : Incidence, concomitant injuries, course and outcome. Unfallchirurg 119(12): 1023-1030, 2016.

13 Cannon RM, Smith JW, Franklin GA, Harbrecht BG, Miller FB and Richardson JD: Flail chest injury: Are we making any progress? Am Surg 78(4): 398-402, 2012.

14 Velmahos GC, Vassiliu P, Chan LS, Murray JA, Berne TV and Demetriades D: Influence of flail chest on outcome among patients with severe thoracic cage trauma. Int Surg 87(4): 240244, 2002.

15 de Moya MA, Manolakaki D, Chang Y, Amygdalos I, Gao F, Alam HB and Velmahos GC: Blunt pulmonary contusion: Admission computed tomography scan predicts mechanical ventilation. J Trauma 71(6): 1543-1547, 2011.

16 Cappello M, Yuehua C and De Troyer A: Rib cage distortion in a canine model of flail chest. Am J Respir Crit Care Med 151(5): 1481-1485, 1995.

17 Collins J: Chest wall trauma. J Thorac Imaging 15(2): 112-119, 2000.

18 Kilic D, Findikcioglu A, Akin S, Akay TH, Kupeli E, Aribogan A and Hatipoglu A: Factors affecting morbidity and mortality in flail chest: Comparison of anterior and lateral location. Thorac Cardiovasc Surg 59(1): 45-48, 2011.

19 Livingston DH, Shogan B, John P and Lavery RF: Ct diagnosis of rib fractures and the prediction of acute respiratory failure. $\mathrm{J}$ Trauma 64(4): 905-911, 2008.

20 Chien CY, Chen YH, Han ST, Blaney GN, Huang TS and Chen $\mathrm{KF}$ : The number of displaced rib fractures is more predictive for complications in chest trauma patients. Scand J Trauma Resusc Emerg Med 25(1): 19, 2017.

21 Bulger EM, Arneson MA, Mock CN and Jurkovich GJ: Rib fractures in the elderly. J Trauma 48(6): 1040-1046; discussion 1046-1047, 2000.

22 Gennarelli TA, Wodzin E and Medicine AftAoA: Abbreviated injury scale 2005: Update 2008. Association for the Advancement of Automotive Medicine: Barrington, 2008.

23 Liman ST, Kuzucu A, Tastepe AI, Ulasan GN and Topcu S: Chest injury due to blunt trauma. Eur J Cardiothorac Surg 23(3): 374-378, 2003.

24 Pape HC, Remmers D, Rice J, Ebisch M, Krettek C and Tscherne H: Appraisal of early evaluation of blunt chest trauma: 
Development of a standardized scoring system for initial clinical decision making. J Trauma 49(3): 496-504, 2000.

25 Battle CE and Evans PA: Predictors of mortality in patients with flail chest: A systematic review. Emerg Med J 32(12): 961-965, 2015.

26 Baker SP, O'Neill B, Haddon W Jr. and Long WB: The injury severity score: A method for describing patients with multiple injuries and evaluating emergency care. J Trauma 14(3): 187196, 1974

27 Mutschler M, Nienaber U, Munzberg M, Wolfl C, Schoechl H, Paffrath T, Bouillon B, Maegele $M$ and TraumaRegister DGU: The shock index revisited - a fast guide to transfusion requirement? A retrospective analysis on 21,853 patients derived from the traumaregister dgu. Crit Care 17(4): R172, 2013.

28 Marshall JC, Cook DJ, Christou NV, Bernard GR, Sprung CL and Sibbald WJ: Multiple organ dysfunction score: A reliable descriptor of a complex clinical outcome. Crit Care Med 23(10): 1638-1652, 1995.
29 Huber S, Biberthaler P, Delhey P, Trentzsch H, Winter H, van Griensven M, Lefering R, Huber-Wagner S and Trauma Register DGU: Predictors of poor outcomes after significant chest trauma in multiply injured patients: A retrospective analysis from the German Trauma Registry (Trauma Register DGU(R)). Scand J Trauma Resusc Emerg Med 22: 52, 2014.

30 Kleber C, Giesecke MT, Tsokos M, Haas NP and Buschmann CT: Trauma-related preventable deaths in berlin 2010: Need to change prehospital management strategies and trauma management education. World J Surg 37(5): 1154-1161, 2013.

Received September 11, 2018

Revised October 5, 2018

Accepted October 8, 2018 